Document downloaded from:

http://hdl.handle.net/10251/81561

This paper must be cited as:

Pérez-Molina, Al.; Díaz-García, P.; Gisbert Soler, V. (2016). Análisis discriminante aplicado a las PYMEs productivas españolas que realizan proyectos de I+D+i. Dyna Management. 4(1):1-12. doi:10.6036/MN7925.

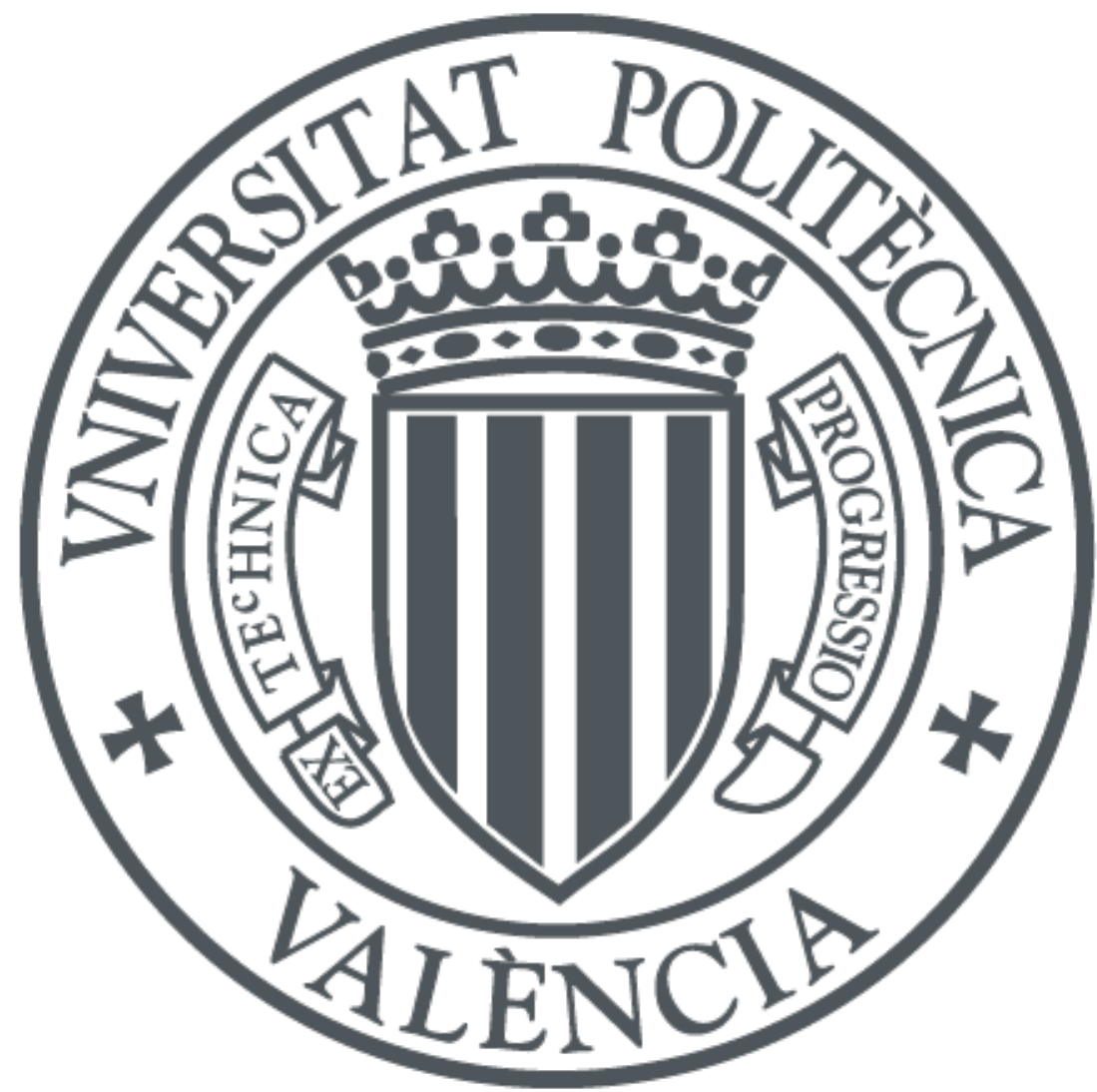

The final publication is available at

http://dx.doi.org/10.6036/MN7925

Copyright Federación de Asociaciones de Ingenieros Industriales de España (FAIIE)

Additional Information 


\title{
ANÁLISIS DISCRIMINANTE APLICADO A LA PYMES PRODUCTIVAS ESPAÑOLAS QUE REALIZAN PROYECTOS DE $\mathbf{I}+\mathbf{D}+\mathbf{i}$
}

\author{
Ana Isabel Pérez-Molina1 ${ }^{1}$ Pablo Díaz-García² y Víctor Gisbert-Soler ${ }^{3}$
}

1 Universitat Politècnica de Valencia. Departamento de Estadística e Investigación Operativa Aplicadas y Calidad. Plaza Ferrándiz y Carbonell, s/n 03801 Alcoy (Alicante), España. anpemo@eio.upv.es

2 Universitat Politècnica de Valencia, Departamento de Ingeniería Textil y Papelera. Plaza Ferrándiz y Carbonell, s/n 03801 Alcoy (Alicante), España.

3 Universitat Politècnica de Valencia. Departamento de Estadística e Investigación Operativa Aplicadas y Calidad. Plaza Ferrándiz y Carbonell, s/n 03801 Alcoy (Alicante), España.

Received: -- Accepted: - DOI: 10.6036 (To be completed by Editor)

\section{DISCRIMINANT ANALYSIS APPLIED TO SPANISH PRODUCERS SMES PERFORMING PROJECTS R\&D\&I}

\section{ABSTRACT:}

Business innovation based on research, development and technological innovation plays a key role as a driver of business competitiveness and socioeconomic growth.

Technological innovation studies usually focus on the analysis of expenditures. However bilateral or multilateral relations are not discussed, even though it is known that in the microeconomic field, the relationship between R\&D\&l and competitiveness reveals complex and not reducible to a simple linear relationship. Numerous quantitative studies related to SMEs that do Research, Technological Development and Technological Innovation (R\&D\&I) exist, but if a company decides to start innovating, there are no references on structures, organizations, performances, etc. available in companies that already innovate.

It has a systematic self-analysis to study the Spanish productive SMEs performing R\&D\&l, focusing on factors of company projects and mixed, which is based on an interview for SME managers analysis of projects based on Binding Motivated Reports (BMR) and an assessment of the main data presented in the Trade Register. As justified in developing systematic, the analyzed entities have a common characteristic and it is that they have achieved at least one IMV in any of the annualities worked.

In order to model the sample of companies and projects there has been several discriminant analysis, which allow to classify a set of cases into different groups from the variables that make up the models obtained.

Keywords: innovative companies, organizational model, project management R\&D\&I, discriminant analysis.

\section{RESUMEN:}

La innovación empresarial basada en la investigación, el desarrollo y la innovación tecnológica juega un papel fundamental como elemento impulsor de la competitividad empresarial y del crecimiento socioeconómico. (1)

Generalmente los estudios sobre innovación tecnológica se centran en el análisis de los gastos. Sin embargo no se analizan las relaciones bilaterales ni multilaterales, aun cuando se conoce que en el terreno microeconómico, la relación entre $1+D+i$ y competitividad se revela compleja y no reductible a una simple relación lineal. Coexisten diversos estudios cuantitativos relacionados con las pequeñas y medianas empresas que hacen Investigación, Desarrollo Tecnológico e Innovación Tecnológica $(1+D+i)$, pero si una sociedad decide empezar a innovar no dispone de referencias sobre cuáles son las estructuras, organizaciones, funcionamientos, etc. que disponen las empresas que ya innovan. (2-9)

Se dispone de una sistemática de análisis propia para estudiar a las pymes productivas españolas que realizan proyectos de I+D+i, centrada en factores de empresa, de proyectos y mixtos, que se sustenta en una entrevista para los directivos de las pymes, un análisis de los proyectos basado en los Informes Motivados Vinculantes (IMV) y una valoración de los principales datos presentados en el Registro Mercantil. (10) Utilizando dicha sistemática, se modeliza una muestra de 80 pymes productivas. Tal y como se justifica en el desarrollo de la sistemática, las entidades analizadas tienen una característica común y es que han conseguido al menos un IMV, en alguna de las anualidades trabajadas.

Con el objetivo de modelizar la muestra de empresas y proyectos se han realizado diversos análisis discriminantes, los cuales permiten clasificar en diferentes grupos un conjunto de casos a partir de las variables que forman los modelos obtenidos.

Palabras clave: empresas innovadoras, modelo organizacional, gestión de proyectos de I+D+i, análisis discriminante. 
Economía del cambio tecnológico

Proyectos de $1+D+i$

\section{1.- INTRODUCCIÓN}

El papel de la innovación y el desarrollo tecnológico sobre la competitividad de las empresas, el bienestar de la sociedad y el progreso de los países ha despertado el interés de muchos investigadores de diferentes ámbitos que han realizado estudios diversos sobre los factores que afectan a la innovación. El gran número de estudios existentes sobre el tema, y el hecho de que haya sido tratado desde diferentes disciplinas y con distintas metodologías, han provocado que en la actualidad, la literatura sea extensa y compleja. En cambio, no existen demasiados trabajos empíricos aplicados al caso español que aborden la innovación desde un punto de vista dinámico. (11)

Son varios los autores que han publicado trabajos donde se analizan los patrones de innovación, sobre todo a nivel regional y/o sectorial, destacando las regiones y/o sectores más avanzados tecnológicamente.

Existen estudios que tratan las relaciones existentes entre la I+D, la innovación y los resultados empresariales limitados y centrados en determinados sectores. En estos estudios se analizan los determinantes de la innovación mediante la integración de diversos aspectos como las estrategias, las capacidades internas de la empresa, etc. pero siempre centrado en un solo sector.(12)

Generalmente los estudios sobre innovación tecnológica se centran en el análisis de los gastos. Sin embargo no se analizan las relaciones bilaterales ni multilaterales, aun cuando se conoce que en el terreno microeconómico, la relación entre I+D+i y competitividad se revela compleja y no reductible a una simple relación lineal. (13)

Por dicho motivo se pretenden modelizar las empresas y los proyectos de I+D+i mediante la técnica estadística de análisis discriminante. Para ello se analiza una muestra de 80 empresas de todo el territorio nacional con una metodología de análisis propia, diseñada especialmente para este fin. La característica común de dichas compañías es que han conseguido al menos un IMV del Ministerio de Economía y Competitividad (MINECO), en alguna de las anualidades trabajadas $(2009,2010$ y 2011). En la muestra de 80 empresas se han obtenido 95 proyectos a analizar, entre los cuales se identifican proyectos de Contenido y Primera Ejecución y proyectos de Seguimiento. ${ }^{1}$

Por lo tanto, como hipótesis de partida se considera que se pretende modelizar mediante un análisis discriminante a un conjunto de variables que sean influyentes para determinados aspectos de la innovación en las Pymes españolas.

\section{2.- DESARROLLO}

Se dispone de una sistemática de análisis para analizar a pequeñas y medianas empresas con una característica común: que hayan conseguido al menos un IMV del MINECO, en alguna de las anualidades trabajadas. (10).

Los IMV son informes de proyectos que emite el MINECO y que contienen la calificación de estos en I+D (Investigación y Desarrollo) y/o IT (Innovación Tecnológica) y sus gastos asociados vinculantes para la Agencia Tributaria. Los IMV se basan en el artículo 35 del Real Decreto Legislativo 4/2004, de 5 de marzo, por el que se aprueba el texto refundido de la Ley del Impuesto sobre Sociedades (TRLIS) y surgen para reducir el nivel de incertidumbre y de riesgo ante la administración para solicitar las deducciones fiscales por I+D+i. Para que una empresa consiga el IMV, previamente necesita obtener un certificado de "Proyecto de I+D+i" de una Entidad Acreditada por la Entidad Nacional de Acreditación (ENAC).

La sistemática de análisis utilizada es capaz de estudiar y relacionar factores de empresa, de proyectos y mixtos, tanto a nivel cualitativo como cuantitativo. Está desarrollada en torno a dos ejes principales: (10)

- Análisis personalizado y específico para cada sociedad donde se pretende obtener información específica sobre la situación de cada empresa y de sus proyectos.

\footnotetext{
${ }^{1}$ Se han trabajado las anualidades 2009, 2010 y 2011 ya que a fecha de envío del presente artículo el MINECO está empezando a trabajar la anualidad 2013, pero todavía no se dispone de una masa crítica suficiente para analizar dicha anualidad.
} 
- Análisis generalista de cada compañía basado en datos donde se pretende obtener información general de la empresa y validar parte de la información obtenida en el otro eje.

La sistemática de análisis se basa en una entrevista con los directivos de las pymes, un análisis de los proyectos basado en los IMV y una valoración de los principales datos presentados en el Registro Mercantil. Concretamente, incluye una modelo de cuestionario-entrevista con los siguientes apartados principales: información general, estructura organizativa, recursos humanos y formación, capacidad tecnológica e innovadora, productos y procesos. Por su parte, el estudio detallado de los proyectos de I+D+i que realizan las sociedades se basa en la información existente en los IMV emitidos por el MINECO, como por ejemplo la duración, planificación, estructura de costes, ayudas recibidas, tipo de novedad, etc. Y la valoración de los principales datos presentados al Registro Mercantil tratará aspectos como la evolución de la facturación, los beneficios, la rentabilidad, etc. Cabe destacar que la sistemática ha sido refrendada por un panel de expertos en la materia. (10)

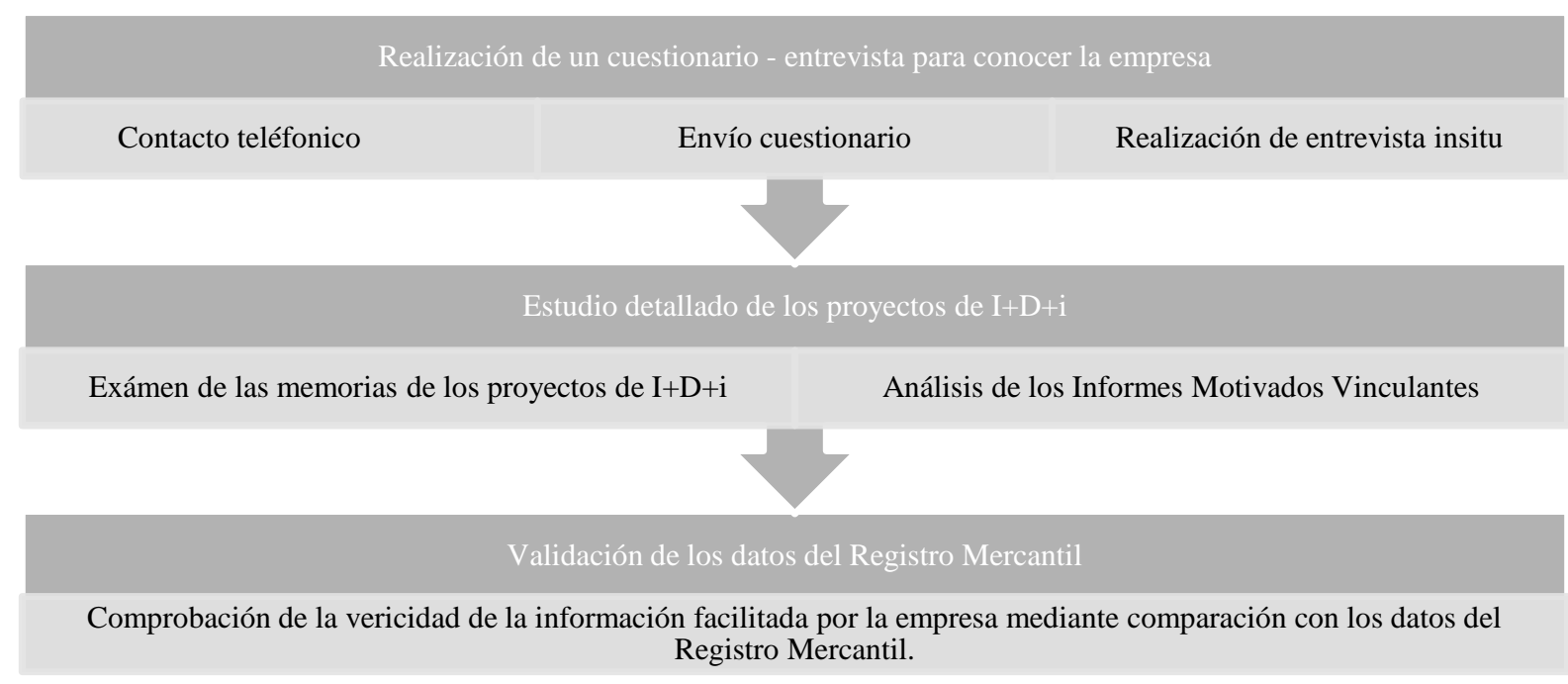

Figura 1. Resumen de la sistemática planteada. Fuente: Elaboración propia a partir de (10).

Se ha aplicado dicha sistemática a la muestra de empresas seleccionadas. Para ello ha sido fundamental el trabajo de campo, basado en la visita a las organizaciones y de los proyectos. Se han analizado tanto variables cuantitativas como cualitativas, y para ello ha sido fundamental cuantificar las cualitativas haciendo valoraciones como por ejemplo bajo, medio, alto. Concretamente, las variables trabajadas han sido las siguientes:

- A nivel de empresa se trabajan las siguientes variables: sector; comunidad autónoma; año de creación; número de trabajadores, facturación y beneficio en los años n, n-1, n-2 y n-5; rentabilidad económica, financiera y endeudamiento en el año n; existencia de un plan estratégico; valoración de la reputación de la sociedad en el mercado; característica destacada de la empresa por los clientes; grado de exportación; gastos de I+D+i en los años n, n-1 y n-2; existencia de suficiente personal en la empresa; previsión de un aumento de personal; clima laboral en la empresa; fomento del trabajo en equipo; adecuación de los mecanismos de comunicación interna; fomento de la innovación desde gerencia; formación del gerente; vinculación entre el gerente y la empresa; existencia de un ambiente adecuado de aprendizaje; valoración de la capacidad tecnológica de la empresa; existencia de un plan estratégico de I+D+i o tecnológico; consideración del nivel de profesionalización de la entidad; número de proyectos de I+D+i gestionados en los años n, n-1, n-2; número de personas dedicadas (total o parcial) a la I+D+i en los años n, n-1 y n-2, especificando en doctores, titulados superiores, titulados 
medios, titulados en formación profesional y no titulados; grado de estructuración del departamento de I+D+i; valoración de la cantidad de recursos humanos dedicados a la $\mathrm{I}+\mathrm{D}+\mathrm{i}$; existencia de sistemas de gestión de la I+D+i; número de publicaciones en revistas especializadas en los últimos 3 años; problemas para financiar la I+D+i; estructuración en la forma de innovar; utilización de herramientas para mejorar la I+D+i; valoración de la novedad de los productos de la empresa; valoración de la importancia de la marca para la introducción de nuevos productos; valoración del estado de mejora / optimización de los procesos existentes en la empresa; valoración del grado de inversiones de los últimos años y frecuencia con la que la empresa presenta proyectos. $(10)^{2}$

- A nivel de variables resultado asociadas a las características de las empresas se trabajan las relaciones entre: lo trabajadores destinados a tareas de $\mathrm{I}+\mathrm{D}+\mathrm{i}$ y los trabajadores totales de la empresa en los años n, n-1 y n-2; el beneficio y la facturación en los años n, n-1, n-2 y n-5; los gastos justificados en I+D+i y el beneficio empresarial en los años n, n-1 y n-2; los gastos justificados en $\mathrm{I}+\mathrm{D}+\mathrm{i}$ y la facturación en los años $\mathrm{n}, \mathrm{n}-1$ y n-2; los gastos justificados en $\mathrm{I}+\mathrm{D}+\mathrm{i}$ y el número de proyectos gestionados en los años n, n-1 y n-2; los gastos de personal aceptados por el MINECO en $\mathrm{I}+\mathrm{D}+\mathrm{i}$ y los gastos justificados en $\mathrm{I}+\mathrm{D}+\mathrm{i}$ en los años $\mathrm{n}, \mathrm{n}-1$ y $\mathrm{n}-2$; los gastos de personal aceptados por el MINECO en I+D+i y el número de personas dedicadas a tareas $\mathrm{I}+\mathrm{D}+\mathrm{i}$ en los años n, n-1 y n-2; el número de Doctores dedicados a tareas de $\mathrm{I}+\mathrm{D}+\mathrm{i}$ y el número de personas dedicadas a tareas de $\mathrm{I}+\mathrm{D}+\mathrm{i}$ en los años n, n-1 y n-2; ídem anterior para Titulados Superiores, Titulados Medios, Titulados en Formación Profesional y No Titulados.

- A nivel de proyecto se trabajan las siguientes variables: código UNESCO; duración prevista y real; presupuesto, diferenciando por anualidades y especificando para I+D y para IT; gasto justificado (diferenciando por anualidades y especificando para I+D y para IT); gasto aceptado por el MINECO (diferenciando por anualidades y especificando para I+D y para IT); gasto aceptado por el MINECO asociado a la partida de personal interno para todo el proyecto (especificando por anualidades y especificando para I+D y para IT); ídem para la partida Universidades y/o Centros de Innovación; ídem para la partida otras colaboraciones externas; ídem para la partida material fungible; ídem para la partida amortización de activos; ídem para la partida otros gastos; valoración de la innovación del proyecto (producto, proceso, nuevo, mejorado sustancialmente); valoración de la novedad del proyecto (objetiva, subjetiva, nacional, internacional, interna, radical, incremental o imitativa), grado de multiobjetividad del proyecto; objetivo estratégico del proyecto; número total de personas dedicadas al proyecto (especificando en los años n, n-1 y n-2 y diferenciando para doctores, titulados superiores, titulados medios, titulados en formación profesional y no titulados); número total de personas dedicadas en exclusiva a la $\mathrm{I}+\mathrm{D}$ en el proyecto (especificando para los años n, n-1 y n-2 e identificando la tipología); participación del gerente en el proyecto; recepción de ayudas públicas para la ejecución del proyecto (especificando en ayudas a fondo perdido y créditos preferentes); prestamos realizados por entidades financieras para el proyecto; valoración de la tecnicidad de las colaboraciones externas de los proyectos; cooperación con otras entidades y protección de los resultados del proyecto mediante derechos de propiedad.

- A nivel de variables resultado asociadas a las características de los proyectos se trabajan las relaciones entre: la duración real del proyecto y la prevista; el gasto justificado y el gasto previsto total; el gasto aceptado por el MINECO en la partida de Personal con respecto al gasto total aceptado por el MINECO; ídem para las partidas de Universidades y/o Centros de Innovación, Colaboraciones Externas, Material Fungible, Amortización de Elementos de Inmovilizado y Otros gastos; número

\footnotetext{
${ }^{2}$ Para el presente estudio se considera que el año n es 2011, el n-1 el 2010 y así sucesivamente.
} 
total de Doctores dedicados al proyecto en función del número de personas totales dedicadas al proyecto; ídem anterior para Titulados Superiores, Titulados Medios, Titulados en Formación Profesional y No Titulados; cantidad prestada por una entidad financiera a interés de mercado en función del gasto ejecutado del proyecto; cantidad recibida en forma de subvención en función del gasto ejecutado del proyecto; y cantidad recibida en forma de crédito preferente en función del gasto ejecutado del proyecto.

Para conseguir modelizar mediante el análisis discriminante a las pymes productivas españolas que realizan proyectos de $\mathrm{I}+\mathrm{D}+\mathrm{i}$ ha sido necesario aplicar la sistemática de análisis comentada líneas arriba a una muestra significativa de empresas; y posteriormente caracterizar dicha muestra con un análisis univariante y bivariante (11) en el que se ha conseguido caracterizar la estructura, la organización, la forma de trabajo, etc. de las sociedades que realizan actividades de I+D+i, para posteriormente aplicar el análisis discriminante descrito en el presente artículo.

Este procedimiento estadístico es una técnica estadística multivariante que permite clasificar y modelar un conjunto de variables cuando la variable dependiente es categórica y las independientes son cuantitativas, e incluso puede funcionar también cuando son categóricas. El propósito de esta técnica es analizar si existen diferencias significativas entre grupos con respecto a un conjunto de variables. Para comprobar que el modelo es bueno debe comprobarse el nivel de significación. Para ello, si el p-valor asociado al estadístico es inferior al nivel de significación (normalmente 0.05) se rechaza la hipótesis de igualdad entre los grupos, de manera que se puede concluir que la información aportada por las respectivas funciones discriminantes que forman el modelo es, desde un punto de vista estadístico, significativa. [14]

Teniendo en cuenta que se trabaja con una población finita, y que en el presente análisis se trabajan las anualidades 2009, 2010 y 2011; al analizar 80 empresas, se obtiene un error muestral del 8,4\% trabajando con un nivel de confianza del $90 \%$, y del $10 \%$ trabajando con un nivel de confianza del $95 \%$. Por lo tanto, una muestra de 80 empresas se considera estadísticamente significativa y suficiente para realizar el estudio.

Consecuentemente, se ha aplicado la sistemática de análisis a una muestra de 80 empresas, la cual es representativa a nivel geográfico y sectorial.

Se ha completado la información planteada de cada una de las empresas y proyectos, y posteriormente se han trabajado los datos obtenidos. Para ello se han utilizado los programas estadísticos IBM SPSS Statistics 22 y Statgraphics X64. Concretamente, se han procesado los datos mediante diversos análisis discriminantes.

\section{3.- RESULTADOS}

A continuación se muestran los principales resultados obtenidos a lo largo del estudio. Concretamente se exponen los diferentes modelos obtenidos en base a los análisis discriminantes realizados. Un modelo de este tipo permite clasificar en diferentes grupos a la muestra estudiada en función del valor que toman las variables que forman el modelo. Es decir, si por ejemplo, se pretende clasificar la muestra de empresas en función de si han realizado uno, dos o tres proyectos; el modelo discriminante ofrece dos funciones que dependerán de diversas variables, pero no tendrá en cuenta la propia variable de interés, que en este caso es el número de proyectos. En función del valor que adoptan las variables que forman el modelo, se podrán clasificar a las empresas en un sitio u otro.

Se ha considerado adecuado escoger ciertas variables y estudiarlas mediante la técnica del Análisis Discriminante, tal y como se muestra a continuación. La forma en la que se trabaja en este documento se basa en escoger una variable de agrupación y realizar el análisis en función de: las variables de empresa; las variables resultado de empresa; las variables de empresa y las variables resultado de empresa conjuntamente; las variables de proyecto; las variables resultado de proyecto; y las variables de proyecto junto con las variables resultado de proyecto.

A continuación se presentan los modelos válidos obtenidos para las variables analizadas. Un modelo se considera válido cuando a nivel estadístico se puede garantizar que es capaz de discriminar a la muestra en los diferentes grupos 
determinados $^{3}$ y cuando el porcentaje de clasificación correcta del modelo es superior al $70 \%$. Para una misma variables puede haber uno o más modelos válidos.

\section{1.- ANÁLISIS DISCRIMINANTE DEL NÚMERO DE PROYECTOS}

Se han obtenido cuatro modelos capaces de discriminar en función del número de proyectos gestionados por las empresas.

Modelo 1. Basado en variables de empresa:

- $\quad$ Número de Doctores dedicados (total o parcial) a I+D+i en el 2011.

- $\quad$ Número de Doctores dedicados (total o parcial) a I+D+i en el 2010.

- $\quad$ Número de Titulados Medios dedicados (total o parcial) a I+D+i en el 2009.

- Número de Titulados Formación Profesional dedicados (total o parcial) a I+D+i en el 2011.

- Número de Titulados Formación Profesional dedicados (total o parcial) a I+D+i en el 2009.

- Nivel de estructuración del departamento de I+D+i.

- Existencia de sistemas de gestión de la I+D+i.

- Número de publicaciones en revistas especializadas en los últimos 3 años.

- Duración media real de los proyectos.

Porcentaje de clasificación correcta: $98,8 \%$.

Modelo 2. Basado en variables de proyecto:

- Gastos aceptados por el MINECO en la partida de Universidad / Centro investigación en I+D del ejercicio 2011 y 2009.

- Gastos aceptados por el MINECO en la partida de Universidad / Centro investigación en I+D del ejercicio 2009.

- Gastos aceptados por el MINECO en la partida Colaboraciones Externas en I+D en el año 2011.

- Gastos aceptados por el MINECO en la partida Otros Gastos en IT en el año 2009.

- Grado de multiobjetividad del proyecto.

- Número de Doctores dedicados al proyecto en total.

- Número de Titulados en Formación Profesional dedicados al proyecto en total.

- Número de personas dedicadas en exclusiva a I+D en todo el proyecto.

- Valoración del grado de evidencias del proyecto.

Porcentaje de clasificación correcta: $84,2 \%$.

Modelo 3. Formado por variables resultado de proyecto:

- $\%$ Gastos aceptados por el MINECO en la partida Otros Gastos / Gasto total aceptado por el MINECO.

- $\%$ Doctores dedicados al proyecto / Personas dedicadas al proyecto.

- $\%$ Titulados Medios dedicados al proyecto / Personas dedicadas al proyecto.

Porcentaje de clasificación correcta: $71,6 \%$.

Modelo 4. Formado por variables de proyecto y resultado de proyecto:

\footnotetext{
${ }^{3}$ Para comprobar que el modelo es bueno debe comprobarse el nivel de significación. Para ello, si el p-valor asociado al estadístico es inferior al nivel de significación (normalmente 0.05) se rechaza la hipótesis de igualdad entre los grupos, de manera que se puede concluir que la información aportada por las respectivas funciones discriminantes que forman el modelo es, desde un punto de vista estadístico, significativa. Es decir, cuando el p-valor es menor que 0,05 se considera que el modelo es estadísticamente significativo.
} 
- Gastos aceptados por el MINECO en la partida Otros Gastos en I+D+i del año 2009 y en IT de la anualidad 2009.

- Número total de Doctores, Titulados Medios y Titulados en Formación Profesional dedicados al proyecto.

- Número de personas dedicadas en exclusiva a I+D en todo el proyecto.

- ¿El proyecto ha recibido ayudas públicas?

- Valoración del grado de evidencias del proyecto.

- Inversiones en elementos de inmovilizado material e intangible.

- \% Doctores dedicados al proyecto / Personas dedicadas al proyecto.

Porcentaje de clasificación correcta: $88,4 \%$.

\section{2.- ANÁLISIS DISCRIMINANTE DE LA CALIFICACIÓN DE LOS PROYECTOS}

Se han logrado dos modelos válidos capaces de discriminar en función de la calificación de los proyectos.

Modelo 1. Formado por variables de proyecto:

- Código UNESCO del proyecto.

- Tipo de novedad (producto, proceso o mixta; objetiva o subjetiva; nacional, internacional o interna; radical, incremental o imitativa).

- Participación del gerente en el proyecto.

Porcentaje de clasificación correcta: 92,6\%.

Modelo 2. Basado en variables de proyecto y resultado de proyecto:

- Código UNESCO del proyecto.

- Tipo de novedad (objetiva o subjetiva; nacional, internacional o interna; radical, incremental o imitativa).

- Grado de multiobjetividad del proyecto.

- Participación del gerente en el proyecto.

- Valoración del grado de evidencias del proyecto.

- \% Gasto aceptado por el MINECO en la partida de Personal / Gasto total aceptado por el MINECO.

- \% Gasto aceptado por el MINECO en la partida Colaboraciones Externas / Gasto total aceptado MINECO.

Porcentaje de clasificación correcta: 95,8\%.

\section{3.- ANÁLISIS DISCRIMINANTE DEL FOMENTO DE LA INNOVACIÓN DESDE LA GERENCIA}

Se ha alcanzado un modelo válido capaz de discriminar en función del grado de fomento de la innovación desde la gerencia.

Modelo 1. Centrado en variables de proyecto:

- $\quad$ Presupuesto en IT del proyecto en la anualidad 2009.

- Gasto justificado en el proyecto en IT en el año 2010.

- Gasto aceptado por el MINECO en las siguientes partidas y años: Fungibles en I+D+i en los años 2009, 2010, 2011; Amortización de Activos en I+D+i en 2009 y en I+D en 2011; Colaboraciones Externas en I+D en 2010 y en IT 2009.

- Valoración del grado de evidencias del proyecto.

Porcentaje de clasificación correcta: $75,8 \%$. 


\section{4.- ANÁLISIS DISCRIMINANTE DE LA CAPACIDAD TECNOLÓGICA DE LA EMPRESA}

Se ha conseguido un modelo válido capaz de discriminar en función de la capacidad tecnológica de la empresa.

Modelo 1. Formado por variables de empresa:

- Año creación de la empresa

- Existencia de plan estratégico.

- Valoración de la reputación de la empresa en el mercado.

- Fomento del trabajo en equipo.

- Nivel de profesionalización de la empresa.

- Utilización de herramientas para mejorar la I+D+i.

- Aprovechamiento de las ayudas públicas.

Porcentaje de clasificación correcta: 78,8\%.

\section{5.- ANÁLISIS DISCRIMINANTE DEL NIVEL DE PROFESIONALIZACIÓN DE LA EMPRESA}

Se han obtenido dos modelos válidos capaces de discriminar en función del nivel de profesionalización de la empresa.

Modelo 1. Formado por variables de proyecto:

- Cantidad aceptada por el MINECO en IT en los años posteriores a 2011.

- Novedad tecnológica nacional, internacional o interna.

- Número total de Titulados Superiores dedicados al proyecto.

- Valoración del grado de evidencias del proyecto.

Porcentaje de clasificación correcta: 71,6\%.

Modelo 2. Basado en variables de proyecto y resultado de proyecto:

- Cantidad aceptada por el MINECO en IT en los años posteriores a 2011.

- Novedad tecnológica nacional, internacional o interna.

- Número total de Titulados Superiores dedicados al proyecto.

- Valoración del grado de evidencias del proyecto.

- Porcentaje de desviación temporal del proyecto.

Porcentaje de clasificación correcta: $73,7 \%$.

\section{6.- ANÁLISIS DISCRIMINANTE DE LA EXISTENCIA DEL PLAN ESTRATÉGICO DE I+D+I O TECNOLÓGICO}

Se han alcanzado dos modelos válidos capaces de discriminar en función de la existencia del plan estratégico de I+D+i o tecnológico.

Modelo 1. Formado por variables de empresa:

- $\quad$ Facturación del año 2006.

- Existencia de plan estratégico.

- Número de Titulados Superiores dedicados (total o parcial) a I+D+i en el 2011 y en el 2010.

- $\quad$ Existencia de sistemas de gestión de la I+D+i.

Porcentaje de clasificación correcta: 75,0\%. 
Modelo 2. Formado por variables de proyecto:

- Cantidad justificada en IT en los años posteriores a 2011.

- Gastos aceptados por el MINECO en la partida Colaboraciones Externas en I+D en el año 2010 y en Fungibles en I+D en todo el proyecto.

- Número total de Titulados Superiores dedicados al proyecto.

- Valoración del grado de evidencias del proyecto.

Porcentaje de clasificación correcta: 75,8 \%.

\section{7.- ANÁLISIS DISCRIMINANTE DE LA PARTICIPACIÓN DE UNIVERSIDADES Y CENTROS TECNOLÓGICOS}

Se han conseguido tres modelos válidos capaces de discriminar en función de la participación de Universidades y Centros tecnológicos en los proyectos.

Modelo 1. Está formado por variables de empresa:

- Rentabilidad financiera de la empresa del año 2011.

- Existencia de plan estratégico.

- Fomento del trabajo en equipo.

- Formación del gerente.

- Vinculación del gerente con la empresa.

- Número de Titulados Medios dedicados (total o parcial) a I+D+i en el 2011 y de Titulados en Formación Profesional dedicados a la I+D+i en el 2009.

- Utilización de herramientas para mejorar la I+D+i.

- Duración media real de los proyectos.

Porcentaje de clasificación correcta: $87,4 \%$.

Modelo 2. Formado por variables de proyecto:

- Duración prevista del proyecto.

- Gastos aceptados por el MINECO en la partida Amortización Activos en I+D+i en las anualidades 2009, 2010, 2011 y en Colaboraciones externas en IT en el año 2009.

- Número total de Titulados Medios dedicados al proyecto 2011, y de Titulados en Formación Profesional dedicados al proyecto en 2009.

- Inversiones en elementos de inmovilizado material e intangible.

Porcentaje de clasificación correcta: 78,9\%.

Modelo 3. Formado por todo tipo de variables:

- Gastos aceptados por el MINECO en la partida Amortización Activos en I+D+i en el año 2010 y en Fungibles en IT en la anualidad 2009.

- Tipo de novedad (producto, proceso o mixta).

- Número de Titulados en Formación Profesional dedicados al proyecto en la anualidad 2009.

- Número de personas dedicadas en exclusiva a I+D en el proyecto en el año 2009.

- ¿El proyecto ha recibido ayudas públicas?

- Cambios en la calificación realizados por el MINECO.

- Calificación inicial dada por la empresa. 
- \% Gasto aceptado por el MINECO en la partida Colaboraciones Externas / Gasto total aceptado por el MINECO.

- \% Cantidad recibida en forma de crédito preferente / Gasto ejecutado total.

- Resultado de la empresa en 2009.

- Clima laboral en la empresa.

Porcentaje de clasificación correcta: $74,7 \%$.

\section{CONCLUSIONES}

Este trabajo ha conseguido profundizar en el conocimiento sobre las pymes productivas españolas que realizan proyectos de I+D+i, ya que se han obtenido modelos discriminantes capaces de relacionar diversas variables. Para ello se ha estudiado la manera que las pymes tienen de innovar, analizando los parámetros que caracterizan a la empresa, estudiando los patrones de innovación y evaluando los resultados.

Con los análisis discriminantes se han determinado tendencias para que las pymes españolas puedan adoptar ciertas estructuras para conseguir incluir la innovación dentro de la organización. Ya que, este trabajo ofrece a las pymes diversos modelos que mezclan variables cuantitativas y cualitativas, a nivel de empresa y proyectos. Seguidamente se muestran las conclusiones obtenidas de los resultados presentados líneas arriba.

\section{1.- ANÁLISIS DISCRIMINANTE DEL NÚMERO DE PROYECTOS}

Se considera que los modelos más adecuados son el 1, el 4 y el 2, respectivamente. Destaca que se puede discriminar el número de proyectos tanto con variables de empresa como con variables de proyecto. El modelo 3 se descarta por estar de manera indirecta incluido en el modelo 4 y ser este más completo. Tras analizar los modelos, se observa que las siguientes variables son las más influyentes:

- Número de Doctores dedicados a I+D+i.

- Número de Titulados Medios dedicados a I+D+i.

- Número Titulados en Formación Profesional dedicados a I+D+i.

- Número de personas dedicadas en exclusiva a I+D.

- Grado de evidencias del proyecto.

- Gasto aceptado por el MINECO en la partida Universidad / Centro investigación en I+D.

- Gasto aceptado por el MINECO en la partida Colaboraciones Externas en I+D.

- Gasto aceptado por el MINECO en la partida Otros Gastos en I+D+i

- Nivel de estructuración del departamento de I+D+i.

- Existencia de sistemas de gestión de la I+D+i.

\section{2.- ANÁLISIS DISCRIMINANTE DE LA CALIFICACIÓN DE LOS PROYECTOS}

Se considera que ambos modelos son adecuados para determinar la calificación de los proyectos, siendo el modelo 2 más completo. Tras estudiar ambos modelos, se observa que las variables más influyentes son:

- Código UNESCO del proyecto. 
- Tipo de novedad (objetiva o subjetiva; nacional, internacional o interna; radical, incremental o imitativa).

- Participación del gerente en el proyecto.

\section{3.- ANÁLISIS DISCRIMINANTE DEL FOMENTO DE LA INNOVACIÓN DESDE LA GERENCIA}

Únicamente se ha obtenido un modelo válido.

\section{4.- ANÁLISIS DISCRIMINANTE DE LA CAPACIDAD TECNOLÓGICA DE LA EMPRESA}

Solamente se ha conseguido un modelo válido.

\section{5.- ANÁLISIS DISCRIMINANTE DEL NIVEL DE PROFESIONALIZACIÓN DE LA EMPRESA}

Se considera que ambos modelos son adecuados para determinar el nivel de profesionalización de la empresa, ahora bien, el modelo 2 es más adecuado pues tiene implícito el modelo 1 y además añade otra variable y aumenta el porcentaje de clasificación correcta. Las variables más influyentes al discriminar en función del nivel de profesionalización son:

- Novedad tecnológica nacional, internacional o interna de los proyectos.

- Número total de Titulados Superiores dedicados a los proyectos.

- Valoración del el grado de evidencias del proyecto.

\section{6.- ANÁLISIS DISCRIMINANTE DE LA EXISTENCIA DEL PLAN ESTRATÉGICO DE I+D+I O TECNOLÓGICO}

Se considera que los dos modelos obtenidos son adecuados. No se han observado variables comunes en ambos modelos.

\section{7.- ANÁLISIS DISCRIMINANTE DE LA PARTICIPACIÓN DE UNIVERSIDADES Y CENTROS TECNOLÓGICOS}

Todos los modelos son válidos, ahora bien, se considera más oportuno utilizar el modelo 1 o el 2, por estar centrados en variables de empresa o de proyecto y consecuentemente son más fáciles de usar, y además tienen un porcentaje de clasificación correcta más elevado.

Tras estudiar los modelos, se aprecia que las variables más influyentes son las siguientes:

- Número de Titulados Medios dedicados a I+D+i.

- Número de Titulados en Formación Profesional dedicados a I+D+i.

- Número de personas dedicadas en exclusiva a I+D.

- Gastos aceptados por el MINECO en la partida Amortización de Activos.

- Gastos aceptados por el MINECO en la partida Colaboraciones Externas. 
Economía del cambio tecnológico

Proyectos de $1+D+i$

- Duración de los proyectos.

- Recepción de ayudas públicas.

En resumen, se han conseguido modelizar diversos aspectos de las pymes productivas españolas que realizan proyectos de $\mathrm{I}+\mathrm{D}+\mathrm{i}$, consiguiéndose por lo tanto, modelos capaces de asistir a las empresas españolas hacia conseguir incluir la I+D+i en su estrategia empresarial, lo cual generará nuevas posibilidades para la mejora de la competitividad. Es decir, se han conseguido determinar las tendencias de las pymes españolas que realizan proyectos de I+D+i en base a los Informes Motivados Vinculantes. Esta sistematización del proceso de innovación puede considerarse el punto de partida, ahora bien, habrá muchísimas circunstancias, tanto internas como externas, que favorecerán o dificultaran la capacidad de innovar de las organizaciones. Por este motivo, es importante tener en cuenta que la I+D+i no debe gestionarse al margen del entorno de la empresa, pues es esencial valorar las particularidades del mercado y del entorno previamente a abordar un proyecto de $\mathrm{I}+\mathrm{D}+\mathrm{i}$.

Una vez estudiadas las situaciones que describen un entorno innovador y, consecuentemente, fomentan la realización y el desarrollo de proyectos de $\mathrm{I}+\mathrm{D}+\mathrm{i}$, se puede concluir que no existe un perfil general típico de pyme innovadora, pero sí hay una serie de aspectos que benefician o dificultan la obtención de nuevos o mejorados procesos y/o productos.

\section{BIBLIOGRAFÍA}

[1] Ministerio de Economía y Competitividad. "Informes Motivados para deducciones fiscales por actividades de I+D e innovación tecnológica". 2012.

[2] López Mielgo N. Comportamiento innovador en la empresa manufacturera española. Tesis Doctoral, 2004.

[3] Lucendo-Monedero AL, Jordá-Borrell RM. "Escenarios para una estadística sobre innovación de dimensión regional: su aplicación en Andalucía". ECONOMÍA INDUSTRIAL, ISSN 0422-2784, №344, 2002.

[4] Rodeiro-Pazos D, López-Penabad MC. "A innovación como factor clave na competitividade empresarialun estudo empírico en pemes galegas". REVISTA GALEGA DE ECONOMÍA: PUBLICACIÓN INTERDISCIPLINAR DA FACULTADE DE CIENCIAS ECONÓMICAS E EMPRESARIAIS, ISSN 1132-2799, Vol. 16, №. 2, 2007.

[5] Mancebo-Fernández N, Llach - Pagès J, Bikfalvi A. Patrones regionales de comportamiento innovador. Análisis de la encuesta del INE a partir del caso de Cataluña. El comportamiento de la empresa ante entornos dinámicos: XIX Congreso anual y XV Congreso Hispano Francés de AEDEM, Vol. 1, 2007.

[6] ] Madrid-Garre M, Sabater-Sánchez R, Sanz-Valle R. "La I+D en la Comunidad Autónoma de la Región de Murcia". REVISTA MADRI+D, 2008.

[7] Gómez-Vieites A, Calvo-González, JL. "Un análisis de las relaciones entre I+D, innovación y resultados empresariales: el sector de electrónica e informática en España". ECONOMÍA INDUSTRIAL, ISSN 0422-2784, № 376, 2010.

[8] García-Piqueres G. "Capacidad de innovación a nivel sectorial: modelización y evidencia empírica para España". Tesis Doctoral, 2011

[9] Calvo-González, JL. Una caracterización de la innovación tecnológica en los sectores manufactureros españoles: algunos datos. ECONOMÍA INDUSTRIAL, ISSN 0422-2784, №331, 2000.

[10] Pérez-Molina Al, Díaz-García P, Gisbert-Soler, V. "Development of a systematic analysis for the characterization of spanish productive SME performing projects R\&D\&l". International scientific Conference. Innovative solutions for sustainable development of textiles and leather industry. May, 23rd - 24 th, 2014 Oradea/Romania. University of Oradea, 2014. Issn: 1843-813X.

[11] Pérez-Molina Al, Gisbert-Soler, V, Díaz-García P. "Caracterización de pymes productivas españolas que realizan proyectos de I+D+i en base a Informes Motivados Vinculantes". DOI: http://dx.doi.org/10.6036/MN7840. 
[12]. Gómez-Vieites AM, Calvo-González, JL. "Un análisis de las relaciones entre I+D, innovación y resultados empresariales: el sector de electrónica e informática en España. ECONOMÍA INDUSTRIAL, ISSN 0422-2784, № 376, 2010

[13] Zubiaurre-Goena A, Buesa-Blancom. "Patrones tecnológicos y competitividad. Un análisis de las empresas innovadoras en el País Vasco" EKONOMIAZ: REVISTA VASCA DE ECONOMÍA, 1999.

[14] RODRÍGUEZ JAUME, María José; MORA CATALÁ, Rafael. "Análisis discriminante". En: Estadística informática : casos y ejemplos con el SPSS / María José Rodríguez Jaume, Rafael Mora Catalá. Alicante : Publicaciones de la Universidad de Alicante, 2001. ISBN 84-7908-638-6, pp. 159-175 